\title{
'Inside the Box': A Cooperative Game for Co-Creating Energy Efficient Retail Spaces
}

\author{
Elli Nikolaidou ${ }^{1}$, Yang $\mathrm{Yu}^{1}$, Robert Schmidt-III ${ }^{1}$, Andrew Dainty ${ }^{1}$, Malcolm Cook $^{1}$, \\ Ksenia Chmutina ${ }^{1}$, Dashamir Marini ${ }^{2}$ \\ ${ }^{1}$ School of Architecture, Building and Civil Engineering, Loughborough University, Loughborough, \\ UK; ${ }^{2}$ Centre for Sustainable Energy Use in Food Chains, Brunel University London, London, UK
}

\begin{abstract}
Although sustainability is one of the drivers of change in the retail sector, employees still treat energy management as a lower priority compared with other operational tasks. As digital technologies are flourishing, gamification is an emerging method of raising energy awareness, with most examples however targeting individuals, and therefore not supporting teamworking approaches to handling end user building energy demand. As such, combining behavioural incentivisation and technological development is a critical socio-technical challenge within the retail environments. The development of a new cooperative role-playing game that harnesses the participatory character of game theory to boost collegiality and encourage the energy-conscious behaviour of staff in a supermarket located in the UK, is described. By feeding the game with energy simulation results, this can be regarded as a novel synergy between behavioural science and game theory within the field of building energy. Future research will focus on testing the real-world potential of the game to engage retail staff in co-creating energy efficient stores.
\end{abstract}

\section{Introduction}

The retail sector is an increasingly complex environment undergoing continuous changes, driven by sustainability prerequisites, novel technologies and shifting customer preferences (ARUP 2017). In this fast-changing context, businesses will need to change in order to maintain the engagement of different stakeholders and, in particular, of their employees (Reeves and Read 2009). Being part of a study that aims to examine the engagement of different stakeholders (that is, retailers, staff, customers as well as designers) in co-creating energy efficient retail stores, this paper will focus on the engagement of staff in delivering the environmental agenda of their organisation through the completion of everyday actions in their stores. In this context, this paper will cast light on the potential of digital cooperative role-playing games to improve the attitude of retail staff towards energy-conscious behaviour.

\section{Employee engagement in energy efficiency practice}

Employee engagement is one of the principal energy work streams in the retail sector, which aims to guarantee store participation in energy efficiency practice. According to the Sustainable Consumption and Production Action Plan (European Commission 2008), retailers should consider sustainability as an opportunity for their business to grow, compete and innovate while reducing their environmental impact. Even though working towards a sustainable future requires a collective effort (ARUP 2017), employees still treat energy management as a lower priority - compared with other store tasks such as customer service (Christina et al. 2015). In addition to the refurbishment of their stores that can directly increase energy efficiency, retailers thus need to draw attention to the behaviour of their employees and raise their environmental awareness (Galvez-Martos et al. 2013). In particular, retailers must explore new ideas of boosting the personal interest of employees in energy performance. As revealed by interviews with employees (Christina et al. 2015), although management staff may be motivated by financial concerns, junior staff does not commonly get stimulated by possible decreases in store profitability, this creating a gap in motivation.

Teamworking is also a crucial aspect of staff engagement within retail stores, where tasks are often interdependent. Different stakeholders may thus need to interact to resolve problems as they arise by making use of a range of skills (Clegg 2000). These skills refer to the job characteristics of employees and hence to their anticipated behaviours in situations requiring action (Kim et al. 2009). Employees should have the opportunity to cultivate their individual and teamworking skills through training programs that prepare them to deal with real-world tasks (Martin 2005). However, the organisational environmental strategy is not always clear to employees, as they are often reported to treat energy-related tasks as an 'add-on' to their existing job responsibilities (Christina et al. 2015). Retailers must thus firstly develop a culture of environmental awareness and secondly disseminate it to their employees through a training scheme, which is able to ensure the engagement of all staff members and ultimately influence their attitude towards energy management (Davis and Coan 2015).

Adopting a socio-technical approach to establishing and communicating this culture can increase motivation and productivity among staff members, as it can cast light on and convey the skills that are vital for the completion of (interdependent) tasks (Birdi et al. 2008). In other words, such an approach can clarify how different stakeholders should interact with each other to tackle real-world tasks, focusing on three main elements: communication, action and establishment of organisational relationships (van der Heijden et al. 2012). Combining these elements can assist stakeholders in effectively imbedding sustainability in the organisational culture and, in particular, translating what they learn into actionable insights and resolving problems as they emerge (Daft and Weick 1984). 


\section{The role of new digital technologies}

Given the continuous expansion of e-commerce, retailers are expected to secure the future success of their physical stores by creating a unique experience for their customers that harnesses new digital technologies (such as artificial intelligence and virtual reality) (ARUP 2017). However, the future opportunity of retailers to innovate is dependent on the technological literacy of their employees, who will need to be acquainted with nascent technologies to guide shoppers and optimise their experience (Hart et al. 2007). At the same time, employees will have to contribute to the sustainability practice of their store to minimise its energy use and carbon footprint (Schönberger et al. 2013).

Confronting the financial impact of employees' behaviour on organisational operation and profitability, retailers will also have to maximise payoffs from staff training (Cascio 2006). Investing in training is critical to guaranteeing the satisfactory performance of staff, who may alternatively be competent at only limited tasks and hence remain inert in situations requiring action (Ton 2014). A good training is also a key ingredient in improving the job productivity and satisfaction of employees, as it can provide them with a better understanding of their role within the organisation and in particular of their responsibilities and relationships with other employees (Rogers et al. 1994).

Given the gradual transition from non-gamers to today's gamer generation, new technologies and in particular new digital games are anticipated to metamorphose learning in the workplace (Beck and Wade 2006). This digital gamebased learning procedure is an emerging field of research, which encourages learning through play (Squire 2011). In an educational adventure game (as it is most commonly called in the literature), play is considered to be part of the learning procedure (Amory 2001). In more detail, players are provided with 'an artificial environment, with which they must interact in order to solve the problems presented in the game' (Cavallari et al. 1992). These problems may represent (complex) real-world tasks, hence inducing the acquisition of knowledge and development of skills that are required in real life (Bellotti et al. 2009). Gameplay can thus combine fun with learning, there however being a fine line as enjoyment should not distract players from learning (Barzilai and Blau 2014).

A role-playing game can further enhance the effectiveness of digital-based learning, as each player picks a character that represents a (real-world) role identity, also inheriting its set of skills (Hong et al. 2009). That is, each player has to embody a persona, while interacting with other players in order to create their improvised team story (Yee 2006). This cooperative learning interaction assists individuals in maximising their understanding of new information and, subsequently, more effectively performing the tasks that are associated with their role (Wiegmann et al. 1992). At the same time, since in cooperative games each agent has a unique set of skills and each task requires a specific set of skills (Bachrach and Rosenschein 2008), autonomous agents need to collaborate to successfully complete tasks (Chalkiadakis et al. 2010).
Cooperative games can hence improve learning from both cognitive and social perspectives (Squire 2002). Focusing on sustainability, such games can be used as educational tools in order to raise awareness of sustainability issues, there however appearing the need to better evaluate their effectiveness in learning - as this is still an emerging field of research (Stanitsas et al. 2018). Having the potential to engage users and raise their awareness, these games can be used to promote desired real-world energy behaviours (Reeves et al. 2012). Energyville (Chevron 2007), The Sims (Adapted) (Tragazikis and Meimaris 2009), and EnerCities (Knol and De Vries 2011) are a few examples of games that have attempted to raise energy awareness. However, these games target individuals and hence do not support cooperative behaviours. Energy Transition Game (Centre for Systems Solutions 2017) and Energy Safari (Ampatzidou and Gugerell 2018) are multi-player games that integrate multiple real-world roles, thus encouraging participatory design. However, these are board - and not digital - games, while, similarly to the aforementioned examples, they do not zoom in on the energy management of a single building and the behaviour of its occupants. The following question hence remains unanswered: (how) can digital cooperative role-playing games affect human behaviour with respect to building energy efficiency and, in particular, (how) can they motivate retail employees to engage in co-creating energy efficient stores?

\section{'Inside the Box': Developing the game}

To explore this question, a novel digital cooperative roleplaying game was developed as a method for encouraging the energy-conscious behaviour of staff in a supermarket located in the UK. To master the game, players must work together effectively to detect and address behaviours that detrimentally affect the energy performance of the store. The game score is informed by the simulation results that were generated using a detailed energy simulation engine. Working closely with the Energy Team of the company and reviewing former initiatives were critical to building the energy model and determining how its results must be communicated within the game to maximise engagement and learning through play, and ultimately ensure the realworld applicability of the game as a training tool.

\section{Case-study building and energy modelling}

The examined building (figure 1) is a supermarket located in the UK having a total useable floor area of $10600 \mathrm{~m}^{2}$ (including the back of the store), constructed in 2013. It is a timber-frame building with a fully-glazed façade facing the North, and skylights for additional daylight. Being compliant with the design standards of the retail company, its sales space has an open-plan layout that is comprised of 17 'sub-areas' (e.g. frozen, bakery and fresh produce). Adhering to operation standards, its heating setpoint is 19 ${ }^{\circ} \mathrm{C}$ and $18{ }^{\circ} \mathrm{C}$ during the day and night, respectively. As it is a 24-hour store, it is heated constantly throughout the day, with natural gas being its main heating fuel. The air handing unit provides fresh air for occupants, while the cooling setpoint is $24{ }^{\circ} \mathrm{C}$ and $25^{\circ} \mathrm{C}$ during the day and night, respectively. To minimise energy use, LED bulbs are used for both internal and external artificial lighting. 


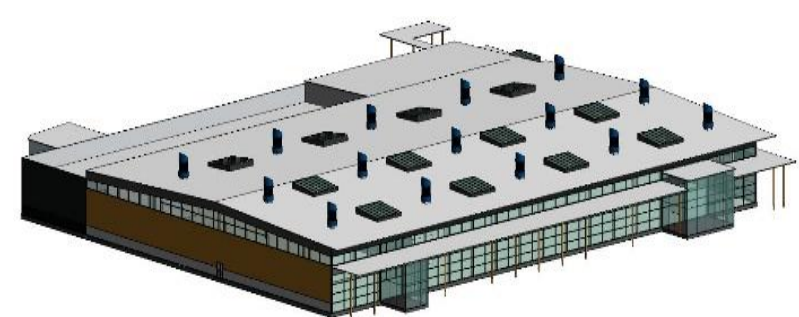

Figure 1: The BIM model of the examined supermarket (source: the retail company (C)).

Figure 2 shows the energy model of the supermarket, built with the Building Information Model (BIM) provided by the retail company. Its form was created in DesignBuilder (DesignBuilder Software Ltd 2018), a popular and userfriendly modelling environment (Attia et al. 2009). The model consists of 31 thermal zones that include the main sales area and secondary spaces at the rear (such as store rooms). After creating the geometry in DesignBuilder, the model was imported into EnergyPlus (U.S. Department of Energy 2016), which is a detailed thermal simulation tool that has been widely reviewed and validated (Henninger and Witte 2004). This is where any additional input data was specified to describe the construction and operation of the building, following the drawings and information provided by the retail company. Note that, with respect to heating, cooling and air conditioning, an ideal loads air system was used, as the interest of this study does not lie in refurbishing the store, but in ameliorating employee engagement in energy efficiency practice.

Creating the energy model of the building had a twofold purpose in this study. Firstly, by running one-parameterat-a-time simulations, it was possible to identify energysaving opportunities that can reduce the carbon footprint of the company. Secondly, it was possible to quantify the influence of these energy saving opportunities, this being vital for the development of the game and, particularly, of its scoring. Working closely with the Energy Team of the company and reviewing the outcomes of past initiatives were crucial to informing the energy model, defining the one-parameter-at-a-time simulations and integrating their results into the game in a comprehensible manner.

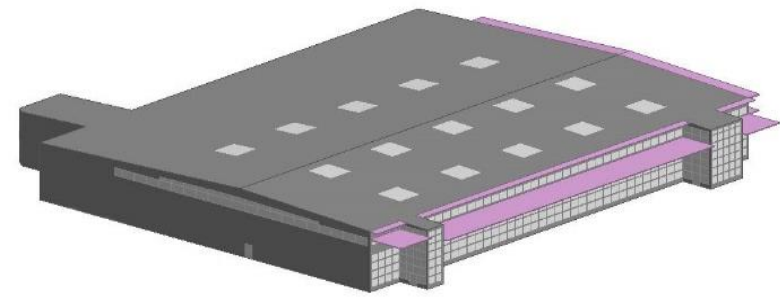

Figure 2: The energy model of the examined supermarket (created in DesignBuilder).

\section{Game design}

Given, on the one hand, the need to change the behaviour of staff with respect to energy and, on the other hand, the potential of games to promote behaviour change (Morford et al. 2014), a cooperative role-playing game called Inside the Box was developed as a means of strengthening staff engagement in energy efficiency practice. The game was developed in Unity (Unity Technologies 2017), an engine that supports scripting in $\mathrm{C \#}$. As the meticulous design of game elements is indispensable to motivating players and stimulating learning (Dondlinger 2007), the description of the game (also summarised in table 1) focuses on its main elements: space; components; mechanics; goals; and rules (Deterding et al. 2011).

Space is a critical element of game design, as this defines the look and feel of the game. The suggested role-playing game takes place in a supermarket and, in particular, in its main sales area. Components are also a determining factor in shaping the context of the game, these referring to its principal parts - i.e. characters and objects. There are four - ready to pick - characters in the game (as illustrated in figure 3), which represent real-world stakeholders/ roles: customer; customer assistant; store manager; and in store technician. Additional avatars can be encountered in the game, these being non-player characters (NPCs) that were programmed to simulate shoppers. Objects represent the selling products, furniture and equipment found in store. Note that, similarly to the layout and construction of the building, furniture and equipment were also informed by the BIM model that was provided by the retail company in order to simulate the real context.

Table 1: Summary of the main elements of the game.

\begin{tabular}{|c|c|c|}
\hline Element & Definition & Description \\
\hline Space & The game environment & The main sales area of a supermarket located in the UK. \\
\hline Components & The parts of the game & $\begin{array}{l}\text { Objects represent selling products, furniture and equipment that can be found in store. } \\
\text { Characters represent real-world stakeholders/ roles (customer, customer assistant, } \\
\text { store manager and technician). }\end{array}$ \\
\hline Mechanics & The actions in the game & $\begin{array}{l}\text { Detect, Collaborate, Fix and Shop are the main actions in the game - as in real life. } \\
\text { These are however restricted by the unique skill set of each character. }\end{array}$ \\
\hline $\operatorname{Goal}(\mathrm{s})$ & How to win the game & $\begin{array}{l}\text { Work together effectively to gather as many points as possible in only } 5 \text { minutes by } \\
\text { performing energy-saving tasks, in parallel with other operational tasks. Make sure } \\
\text { not to rise the energy bill and always keep customers satisfied. }\end{array}$ \\
\hline Rules & How to play the game & $\begin{array}{l}\text { Select a character to start the game. } \\
\text { Detect the (energy-related or operational) problem ( } 10 \text { points). } \\
\text { Collaborate with a team player ( } 10 \text { points), if the problem is not related to your role. } \\
\text { Fix the problem ( } 10 \text { points), if this falls within your area of responsibility. } \\
\text { Shop each product in the shopping list ( } 10 \text { points), but only if you are a customer. }\end{array}$ \\
\hline
\end{tabular}




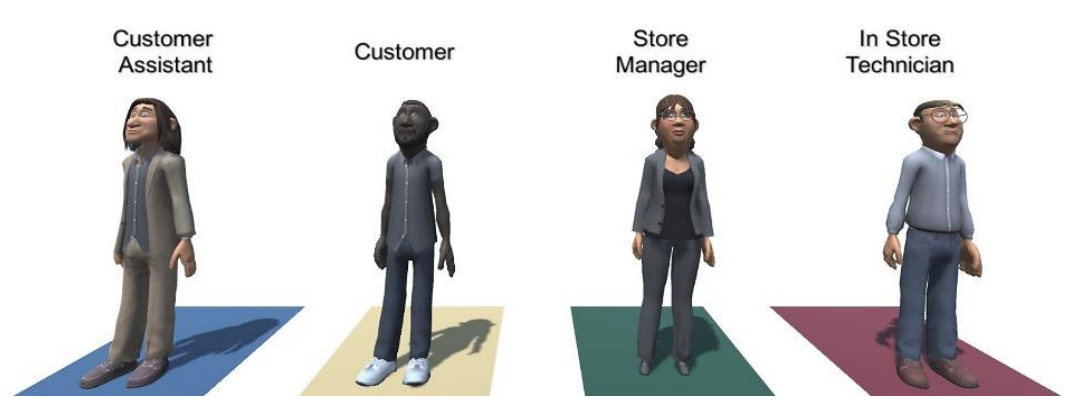

Figure 3: The four characters of the game, which represent real-world roles.

Mechanics define the actions that the players are allowed to perform during gameplay. In the proposed game, there are four principal actions (as demonstrated in figure 4): Detect; Collaborate; Fix; and Shop. Given the importance of mapping game features to real functions in the context of role-playing games (Reeves and Read 2009), these four actions were built based on the real-world responsibilities of stakeholders. Working closely with the Energy Team of the retail company and reviewing the job descriptions of different roles guaranteed the inclusion of the (energyrelated and operational) tasks that each stakeholder needs to perform in real life. These tasks were then reflected on the skills of each character, hence making the game a roleplaying environment that can support learning by doing. Even though this study focuses on employee engagement in energy-related tasks, additional operational tasks were considered in the game. The intent is to more accurately imitate real life, as stakeholders are frequently faced with more than one problem simultaneously and very quickly have to evaluate the trade-offs between acting on one or the other. As an example, a customer assistant may detect an open door on a freezer cabinet, but also an empty shelf that needs to be stocked with merchandise.

The goal of the game is another important element, as this indicates what players should be working towards. To win Inside the Box, (the four) players need to work together effectively to gather as many points as possible in only 5 minutes by completing (character-specific) energy-saving but also operational tasks. They must ensure their actions do not result in increases in energy use (this reflecting the annual energy demand of their store), while maintaining customer satisfaction. This can be achieved by detecting and fixing behaviours that detrimentally affect the energy efficiency of the store and problems that may undermine the experience of customers. Collaborating with a team player is also rewarded and can be the key to winning the game, as each character has their own unique skill set that may not allow them to complete a task individually. The character that represents a customer has an additional task to complete as in real life; that is, to buy the products that are included in the provided shopping list (figure 4).

Finally, rules are vital for guiding players and restricting their actions. After selecting a character, each player must go around the open-plan space of the supermarket to make sure energy efficiency and shopping experience are not undermined. As (energy-related or operational) problems emerge, players have to Detect them. Each problem can be detected only once, allowing the team to gain 10 points.
Given the time restriction, players must quickly make use of their critical thinking to decide whether or not a specific problem falls within their area of responsibility. If this is part of their role, they can then Fix it and gather 10 points for their team. If it is not, they must Collaborate with the team player that is expected to address such a problem in real life, gaining 10 points. In more detail, to gain the 10 points, the player who detected the problem needs to pick (from a dropdown menu, as shown in figure 4) the team player, who will then need to read a message that briefly explains the problem. Finally, if the player impersonates a customer, they will also need to Shop all products in the shopping list, with each product collected awarding the team with 10 points. If more than 25 seconds pass after a problem occurs without it being addressed, the energy bill will rise, or the satisfaction bar will fall, this being subject to the nature of the problem - i.e. if it is related to energy efficiency or customer experience, respectively.

\section{Connecting energy modelling and game design}

After creating and refining the energy model, performing one-parameter-at-a-time experiments revealed that frozen and bakery sub-zones present the greatest opportunities to save energy in the store. In more detail, keeping doors of freezer cabinets open and not turning ovens off when not needed (that is, between bakes and as soon as baking is finished) were proven to be impactful behaviours. At the same time, as revealed by reports of the retail company, these behaviours are frequently observed in supermarkets. Such an observation underlines the importance of making sure equipment will henceforth be operating to the most efficient manner. Since employees affect the operation of equipment, retailers should treat the developed game as a training tool that can convey these impactful behaviours through an engaging and playful experience.

The EnergyPlus (U.S. Department of Energy 2016) model was therefore used to reveal the current energy demand of the store and quantify the impact of commonly-observed behaviours in store, which can adversely affect it. As both the literature review and the discussions with the company highlighted the potential for financial matters to intensify motivation, the simulated energy numbers were translated into monetary values. These values were then included in a C\# script which is run within Unity (Unity Technologies 2017) during gameplay, in order to inform the game score. In this way, players are provided with a quantitative link between their various actions and the energy performance of the store, thus becoming aware of the consequences of their behaviour in real life. 


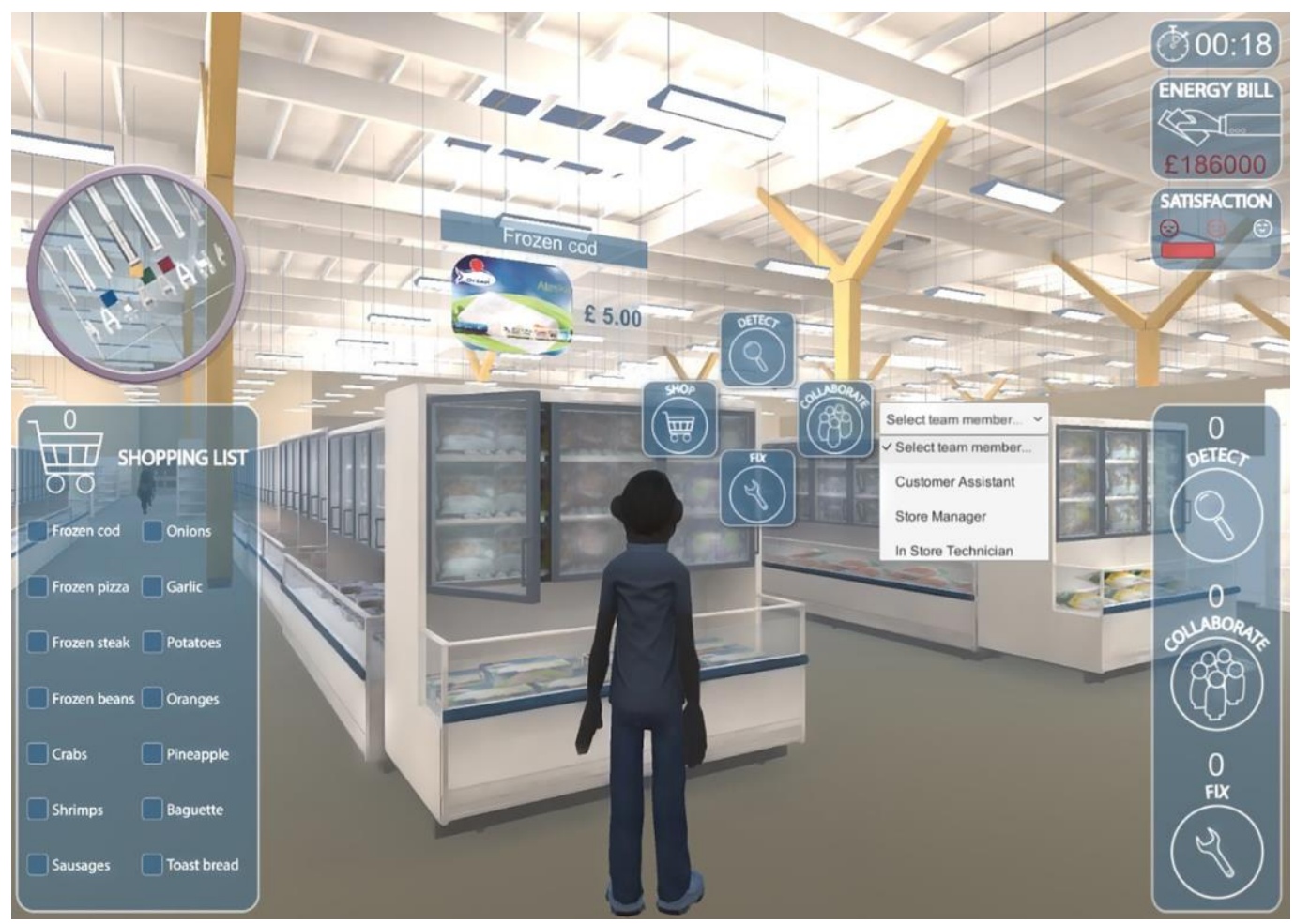

Figure 4: The four main actions in the game: Detect, Collaborate, Fix, and Shop.

In particular, when the game starts, players can find the baseline value of $£ 175000$ at the right top of their screen, which represents the annual operating cost of their store (this including both gas and electricity use). If an energyrelated problem occurs and is not addressed shortly by the players, the energy bill will rise - as would happen in real life. After working closely with the Energy Team of the retail company, it was concluded that, in order to increase employee motivation, the rise in the bill should represent the consequence of the problem on an annual scale and for all equipment (of that particular sub-zone) - i.e. the effect of this behaviour on annual operating cost. In this way, if the problem refers to an open door on a freezer cabinet, the baseline value will be replaced by $£ 186000$ (figure 4) to demonstrate the annual bill rise, in case this behaviour is applied to all doors (as in the case of a single door, the rise would be less than $£ 100$, thus not boosting employee motivation). Similarly, if the problem refers to an oven being on when not necessary, the equivalent value will be $£ 183000$.

Note that these numbers are case-sensitive as they express the increase in operating cost for the examined store and consequently for its specific number of freezers and ovens respectively. When the problem gets addressed by one of the players in the team, the energy bill will fall back to the baseline value. At the same time, an animation will appear to inform the player that they assisted the team in gaining 10 points by completing this action. Additional text at the right side of the screen will present the annual potential savings for this specific zone (frozen or bakery) and hence for the entire store. That is, how much less the store will have to pay annually ( $£ 11000$ or $£ 8000$, respectively), if this energy-related behaviour is adopted. Animation and text aim to make the game an enjoyable experience for the players, while rewarding them for addressing problems in store and providing them with instantaneous feedback on the impact of their action on energy efficiency.

At the end of the game, each player can read the summary, indicating the performance of the whole team, as well as their individual achievements (figure 5). The summary reflects not only their energy-related behaviour, but also the successful (or not) fulfilment of operational tasks that they also need to perform as part of their job role - or, in the case of a customer, the additional act of shopping. In this way, employees learn to stop treating energy-related tasks as an 'add-on' to their existing operational tasks and deal with various problems as they emerge. As in real life, the players that impersonate staff members need to keep a balance between increasing the energy efficiency of the store and improving the experience of shoppers. That is, they need to find a trade-off between the energy bill and the satisfaction bar at the top of their screen, with the latter mirroring the fulfilment of operational tasks (figure 4).

Different stakeholders also learn how they should interact with each other in order to tackle everyday tasks. Having to collaborate when detecting a problem that does not fall within their area of responsibility, employees are trained to work together effectively to address real-life problems. As an example, if the customer assistant detects a water leak, collaborating with the technician is vital for fixing that problem, as this employee is responsible (in real life) for the maintenance of the store. To support motivation and engagement, the action of collaborating with a team member is rewarded in the game - similarly to the actions of detecting, fixing and shopping. 


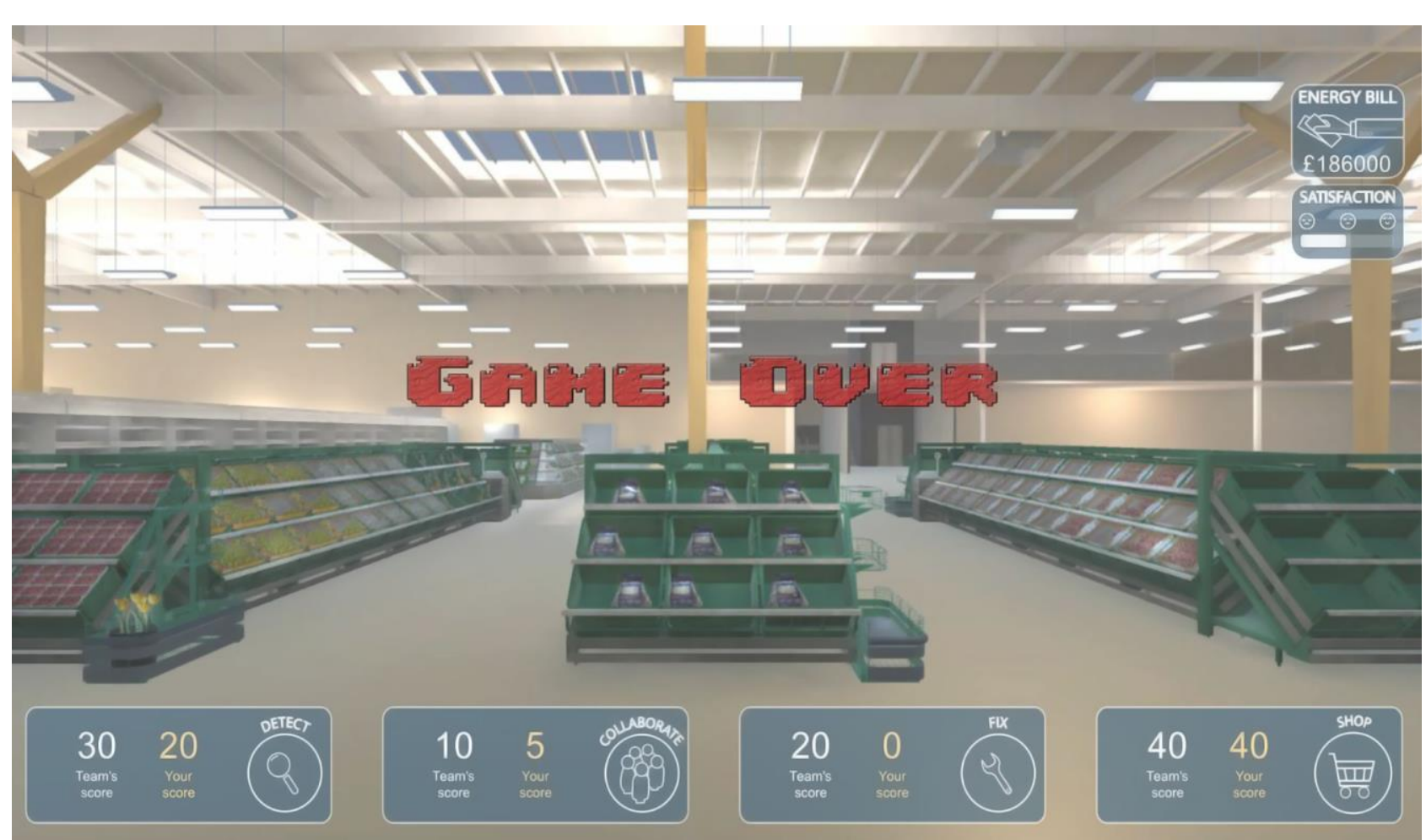

Figure 5: The summary of the game indicating individual and team performance.

\section{Conclusions}

Along with investing in refurbishing their building stock, retailers will also have to invest in increasing the energy awareness of their employees, as their behaviour has the potential to detrimentally affect energy performance. It is hence necessary to embed energy efficiency practice into different roles (e.g. customer assistant, store manager etc.) and clearly disseminate energy-related responsibilities via a comprehensible training scheme. Given the growth of digital technologies and particularly of gamification, the 'learning by doing' philosophy behind games needs to be further investigated as a method of accomplishing energy saving best practice among retail employees, this being the focus area of this study.

The development of a new cooperative role-playing game was described which aims to increase staff engagement in energy efficiency practice within the retail sector. To win the game, players must work together effectively to detect and address common behaviours that detrimentally affect energy efficiency in retail stores. Examining a real-world case study building and working closely with its Energy Team supported the inclusion of real-life, energy-related tasks. Creating the energy model of the store provided a quantitative link between these tasks and predicted energy savings, with frozen and bakery zones having the greatest potential to save energy in store. Other operational tasks were also added to the game to better imitate real practice where several problems emerge simultaneously. Working closely with the Energy Team also informed game design and ensured the comprehensibility of the feedback that the players receive when performing an action. As the game reflects real-world responsibilities and relationships, the players can improve their understanding of the different stakeholder perspectives and interactions in retail stores.

The continual engagement with the retail company during the game development process ensured the usability of the game. Future research will now focus on testing the game in store with the help of different stakeholders. They will evaluate its potential to both support the development of energy-related knowledge and to encourage collaboration around energy-related tasks. This evaluation will uncover how engagement and learning can be enhanced within the game prior to its wide application as a training tool. After refining the game and applying it to train employees, its effectiveness in learning (from both cognitive and social perspectives) will be evaluated through the observation of the attitude of employees towards energy management as well as the energy monitoring of the stores within which it is deployed.

\section{Acknowledgements}

This research is funded by the Engineering and Physical Sciences Research Council (EPSRC). The contribution of the retail company is also gratefully acknowledged.

\section{References}

Amory, A., 2001. Building an educational adventure game: Theory, design, and lessons. Journal of Interactive Learning Research, 12 (2), 249-263.

Ampatzidou, C. and Gugerell, K., 2018. Participatory game prototyping-balancing domain content and playability in a serious game design for the energy transition. CoDesign, 1-16.

ARUP, 2017. Future of retail. 
Attia, S., Beltrán, L., De Herde, A., and Hensen, J., 2009. 'Architect friendly': A comparison of ten different building performance simulation tools. In: BS2009, 11th Conference of International Building Performance Simulation Association, Glasgow, Scotland, July 27-30. 204-211.

Bachrach, Y. and Rosenschein, J.S., 2008. Coalitional skill games. In: Proceedings of the 7th International Joint Conference on Autonomous Agents and Multiagent Systems-Volume 2. 1023-1030.

Barzilai, S. and Blau, I., 2014. Scaffolding game-based learning: Impact on learning achievements, perceived learning, and game experiences. Computers \& Education, 70, 65-79.

Beck, J.C. and Wade, M., 2006. The kids are alright: How the gamer generation is changing the workplace. Harvard Business Press.

Bellotti, F., Berta, R., De Gloria, A., and Primavera, L., 2009. Enhancing the educational value of video games. Computers in Entertainment (CIE), 7 (2), 23.

Birdi, K., Clegg, C., Patterson, M., Robinson, A., Stride, C.B., Wall, T.D., and Wood, S.J., 2008. The impact of human resource and operational management practices on company productivity: A longitudinal study. Personnel Psychology, 61 (3), 467-501.

Cascio, W.F., 2006. The economic impact of employee behaviors on organizational performance. In: America at Work. Springer, 241-256.

Cavallari, B., Heldberg, J., and Harper, B., 1992. Adventure games in education: A review. Australasian journal of educational technology, 8 (2).

Centre for Systems Solutions, 2017. Energy Transition Game [online]. Available from: https://systemssolutions.org/social-simulationsold/energy-transition-game/ [Accessed 6 Dec 2018].

Chalkiadakis, G., Elkind, E., Markakis, E., Polukarov, M., and Jennings, N.R., 2010. Cooperative games with overlapping coalitions. Journal of Artificial Intelligence Research, 39, 179-216.

Chevron, 2007. Energyville [online]. Available from: https://www.chevron.com/ [Accessed 6 Dec 2018].

Christina, S., Waterson, P., Dainty, A., and Daniels, K., 2015. A socio-technical approach to improving retail energy efficiency behaviours. Applied Ergonomics, 47, 324-335.

Clegg, C.W., 2000. Sociotechnical principles for system design. Applied ergonomics, 31 (5), 463-477.

Daft, R.L. and Weick, K.E., 1984. Toward a model of organizations as interpretation systems. Academy of management review, 9 (2), 284-295.

Davis, M.C. and Coan, P., 2015. Organizational change.
In: J. Robertson and J. Barling, eds. The Psychology of Green Organizations. Oxford University Press.

DesignBuilder Software Ltd, 2018. DesignBuilder V5.0.3.007 [online]. Available from: https://www.designbuilder.co.uk/ [Accessed $25 \mathrm{Jul}$ 2018].

Deterding, S., Dixon, D., Khaled, R., and Nacke, L., 2011. From game design elements to gamefulness: defining gamification. In: 15th International Academic MindTrek Conference: Envisioning future media environments, Tampere, Finland, September 28-30. 9-15.

Dondlinger, M.J., 2007. Educational video game design: A review of the literature. Journal of applied educational technology, 4 (1), 21-31.

European Commission, 2008. Sustainable Consumption and Production and Sustainable Industrial Policy Action Plan.

Galvez-Martos, J.L., Styles, D., and Schönberger, H., 2013. Identified best environmental management practices to improve the energy performance of the retail trade sector in Europe. Energy policy, 63, 982-994.

Hart, C., Stachow, G.B., Farrell, A.M., and Reed, G., 2007. Employer perceptions of skills gaps in retail: issues and implications for UK retailers. International Journal of Retail \& Distribution Management, 35 (4), 271-288.

van der Heijden, A., Cramer, J.M., and Driessen, P.P.J., 2012. Change agent sensemaking for sustainability in a multinational subsidiary. Journal of Organizational Change Management, 25 (4), 535559.

Henninger, R.H. and Witte, M.J., 2004. EnergyPlus testing with ANSI/ASHRAE standard 140-2001 (BESTEST). GARD Analytics.

Hong, J.C., Cheng, C.L., Hwang, M.Y., Lee, C.K., and Chang, H.Y., 2009. Assessing the educational values of digital games. Journal of Computer Assisted Learning, 25 (5), 423-437.

Kim, H., Knight, D.K., and Crutsinger, C., 2009. Generation Y employees' retail work experience: The mediating effect of job characteristics. Journal of Business Research, 62 (5), 548-556.

Knol, E. and De Vries, P.W., 2011. EnerCities-A serious game to stimulate sustainability and energy conservation: Preliminary results.

Martin, C.A., 2005. From high maintenance to high productivity: What managers need to know about Generation Y. Industrial and commercial training, 37 (1), 39-44.

Morford, Z.H., Witts, B.N., Killingsworth, K.J., and Alavosius, M.P., 2014. Gamification: the intersection between behavior analysis and game design technologies. The Behavior Analyst, 37 (1). 
Reeves, B., Cummings, J.J., Scarborough, J.K., Flora, J., and Anderson, D., 2012. Leveraging the engagement of games to change energy behavior. In: International Conference on Collaboration Technologies and Systems (CTS), Denver, USA, May 21-25. 354-358.

Reeves, B. and Read, J.L., 2009. Total engagement: How games and virtual worlds are changing the way people work and businesses compete. Harvard Business Press.

Rogers, J.D., Clow, K.E., and Kash, T.J., 1994. Increasing job satisfaction of service personnel. Journal of services Marketing, 8 (1), 14-26.

Schönberger, H., Galvez Martos, J.L., and Styles, D., 2013. Best Environmental Management Practice in the Retail Trade Sector. European Commission JRC Scientific And Policy Reports. Learning from frontrunners.

Squire, K., 2002. Cultural framing of computer/video games. Game studies, 2 (1), 1-13.

Squire, K., 2011. Video Games and Learning: Teaching and Participatory Culture in the Digital Age. Technology, Education-Connections (the TEC Series). ERIC.

Stanitsas, M., Kirytopoulos, K., and Vareilles, E., 2018. Facilitating sustainability transition through serious games: A systematic literature review. Journal of Cleaner Production.

Ton, Z., 2014. The good jobs strategy: How the smartest companies invest in employees to lower costs and boost profits. Houghton Mifflin Harcourt.

Tragazikis, P. and Meimaris, M., 2009. Engaging kids with the concept of sustainability using a commercial video game - A case study. In: Transactions on edutainment III. Springer, 1-12.

U.S. Department of Energy, 2016. EnergyPlus 8.5.0 [online]. Available from: https://energyplus.net/ [Accessed 30 May 2016].

Unity Technologies, 2017. Unity 5.6.1f1 [online]. Available from: https://unity3d.com/ [Accessed 6 Jun 2017].

Wiegmann, D.A., Dansereau, D.F., and Patterson, M.E., 1992. Cooperative learning: Effects of role playing and ability on performance. The Journal of experimental education, 60 (2), 109-116.

Yee, N., 2006. Motivations for play in online games. CyberPsychology \& behavior, 9 (6), 772-775. 